\title{
Alternative Synthesis of 1,8-Difluoroanthracene via the Balz-Schiemann Reaction
}

\author{
Rabih O. Al-Kaysi \\ College of Science and Health Professions, King Saud bin Abdulaziz University for Health Sciences, \\ King Abdullah International Medical Research Center, Ministry of National Guard-Health Affairs, Riyadh 11426, Saudi Arabia
}

Correspondence should be addressed to Rabih O. Al-Kaysi; rabihalkaysi@gmail.com

Received 26 February 2016; Accepted 10 April 2016

Academic Editor: Jinheng Li

Copyright (C) 2016 Rabih O. Al-Kaysi. This is an open access article distributed under the Creative Commons Attribution License, which permits unrestricted use, distribution, and reproduction in any medium, provided the original work is properly cited.

\begin{abstract}
An alternative and improved method for the synthesis of 1,8-difluoroanthracene 5 is described. The precursor 1,8-difluoro9,10-anthraquinone 4 was synthesized by precipitating the water-soluble 1,8-diazonium-9,10-anthraquinone fluoroborate 3 using isopropanol, before thermally decomposing it via the Balz-Schiemann reaction to yield $\mathbf{4}$ in the form of pure sublimed crystals. Compound 5 was later synthesized with $84 \%$ yield by reducing 4 in a $\mathrm{Zn} / \mathrm{NH}_{4} \mathrm{OH}$ mixture. Using isopropanol to quantitatively precipitate water-soluble diazonium fluoroborate salts was also used to synthesize other fluoroanthraquinone derivatives with improved yields.
\end{abstract}

\section{Introduction}

Organofluorine compounds have found extensive use in industry [1] and medicine [2]. Substituting hydrogen for a fluorine atom can enhance the biological activity of some drugs [3-5]. Of particular interest to organic and medicinal chemists are fluoro-aromatic compounds since they are susceptible to "ipso" nucleophilic aromatic substitution more so than $\mathrm{Cl}, \mathrm{Br}$, or I substituents [6]. Fluorine can be substituted with the appropriate nucleophile to form a C-N [7], C-O [8], or C-P [7] bond. In recent years there was extensive interest in fluoro-substituted anthraquinones as precursors for the synthesis of novel antitumor drugs, commercial dyes [9], and more recently photomechanical actuators [10]. Of particular importance is 1,8-difluoro-9,10-anthraquinone 4 [11, 12] and its reduced product 1,8-difluoroanthracene 5 [7, 13-17] which has been used for the synthesis of diphenylphosphino and dimethylamino ligands linked at the 1,8-position of the anthracene backbone. The 1 and 8 carbon positions in 4 or 5 provide a handle onto which a variety of ligands can be attached (via nucleophilic substitution) to form homogenous catalysts [14] or drugs with promising antitumor activity [18]. A quick literature survey reveals that $\mathbf{4}$ was exclusively synthesized using $\mathrm{Cl}-\mathrm{F}$ halogen exchange chemistry. Thermal reaction between the commercially available 1,8-dichloro9,10-anthraquinone and dry $\mathrm{KF}$ under pressure and $230^{\circ} \mathrm{C}$ was the first method described [19] to synthesize 4 . This method was deemed unreliable and could not be reproduced by several authors. More recently Cl-F exchange of 1,8dichloro-9,10-anthraquinone with anhydrous $\mathrm{CsF}$ in anhydrous DMSO $[7,12,20,21]$ became the adopted method. The reagents had to be extensively dried in order to obtain reasonable yields. Tiny amounts of water or failure to obtain bonedry DMSO and CsF reduced the yield to the single digits. The authors of this paper tried to repeat the described literature procedure [7] using anhydrous CsF and DMSO (vacuum distilled then stored over activated molecular sieves $4 \AA$ Á) but failed to yield any product. Reaction of 1,8-dichloro-9,10anthraquinone with flame dried $\mathrm{CsF} / \mathrm{NaF}$ powder at $300^{\circ} \mathrm{C}$ yields a mixture of the mono- and difluorosubstituents that sublime out of the reaction mixture along with a significant amount of the starting material, before achieving complete Cl-F halogen exchange (Scheme 1). This method proved to be tedious and corrosive towards laboratory glassware; also separating the sublimed mixture of products and reactant using column chromatography was impractical and reagent consuming. 


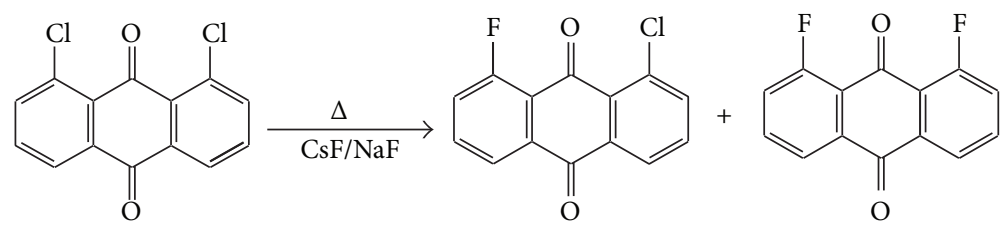

SCHEME 1: Dry mixed thermal Cl-F exchange between 1,8-dichloro-9,10-anthraquinone 7 and CsF/NaF to yield a sublimed mixture of 4 and 6.

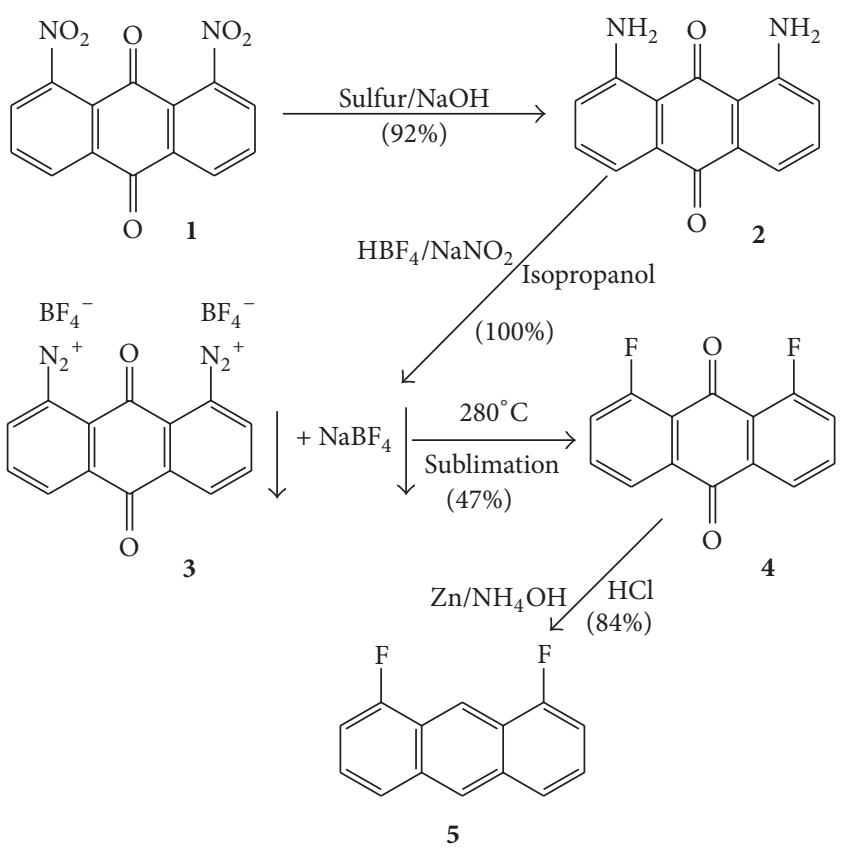

Scheme 2: Synthesis of $\mathbf{4}$ after the coprecipitation of $\mathbf{3}$ followed by thermal decomposition (Balz-Schiemann) to give $\mathbf{4}$. Compound $\mathbf{5}$ is made by reducing 4 in $\mathrm{Zn} / \mathrm{NH}_{4} \mathrm{OH}$ [7] (m.p. = 141-142.5 ${ }^{\circ} \mathrm{C}$, Lit. [7] m.p. $=142^{\circ} \mathrm{C}$ ).

\section{Materials and Methods}

All reagents were purchased from TCI America and used without further purification. For the GC analysis we used a general-purpose column (30 $\mathrm{m}$ long) with a linear velocity flow rate set at $1.2 \mathrm{~mL} / \mathrm{min}$.

For the following synthesis refer to Scheme 2. Commercially available 1,8-dinitro-9,10-anthraquinone was converted to the corresponding 1,8-diamino-9,10-anthraquinone by reducing it with sulfur. Thus elemental sulfur $(5 \mathrm{~g})$ was dissolved in hot aqueous $\mathrm{NaOH}(5 \mathrm{~g}$ in $80 \mathrm{~mL}$ of water) to form the sulfide. 1,8-Dinitro-9,10-anthraquinone $\mathbf{1}$ (3 g) was added to the sulfide solution, along with $50 \mathrm{~mL}$ of ethanol as a cosolvent and stirred for several hours under a blanket of Argon gas till the mixture turns dark red with the formation of a precipitate. 1,8-Diamino-9,10-anthraquinone 2 was suction filtered after diluting the reaction with $500 \mathrm{~mL}$ of cold water. The residue was washed with DI water till the filtrate ran clear. Compound 2 was air-dried to obtain $2.1 \mathrm{~g}(92 \%$ yield) of maroon-red crystals. m.p. $268-270^{\circ} \mathrm{C}$ (Lit. [22] 270$271^{\circ} \mathrm{C}$ ) and $1 \mathrm{H}-\mathrm{NMR}(300 \mathrm{Mhz}, \mathrm{DMSO}-\mathrm{d} 6): \delta 7.86$ (broad singlet, $4 \mathrm{H}), 7.43$ (dd, 2H), 7.35 (d, 2H), and 7.15 (m, 2H). 1,8Diamino-9,10-anthraquinone ( $2 \mathrm{~g}$ ) was stirred with $16 \mathrm{~mL}$ of $50 \% \mathrm{HBF} 4$. Two equivalents of $\mathrm{NaNO}_{2}(1.16 \mathrm{~g})$ were gradually added while stirring and maintaining a temperature $<5^{\circ} \mathrm{C}$. The reaction mixture was stored under a blanket of Argon gas at $0^{\circ} \mathrm{C}$ for an additional 10-18 hours until all the amine was converted to the diazonium salt. The red-brown colored slurry was slowly added to $100 \mathrm{~mL}$ of ice-cold isopropanol and vigorously stirred to precipitate a gray-colored solid composed of a mixture of 3 and $\mathrm{NaBF}_{4}$. The precipitated salts were suction filtered and washed with cold isopropanol to get rid of the excess $\mathrm{HBF}_{4}$ and water, followed by copious amounts of hexanes. The gray-colored powder was gently dried at $45^{\circ} \mathrm{C}$ under reduced vacuum to remove any trace amounts of water. The dry powder was mixed with 5 times its weight in dry sand and packed inside an aluminum foil folded into a puck-shape disk and then pressed flat on the bottom of a $500 \mathrm{~mL}$ beaker. The beaker was covered with aluminum foil and placed on top of a hot plate set at $280^{\circ} \mathrm{C}$ for 15 hours to initiate thermal decomposition of 3 . Product 4 sublimes and condenses on the side of the beaker in the form of orange needle shape crystals (Figure 1). Compound 4 (m.p. 227-229 C, Lit. [12] 228-229 ${ }^{\circ}$; ${ }^{1} \mathrm{H}-\mathrm{NMR}$ (300 Mhz, $\mathrm{CDCl}_{3}$ ) $\delta 8.10(\mathrm{dd}, 2 \mathrm{H}), 7.80(\mathrm{~m}, 2 \mathrm{H})$, and $7.55(\mathrm{~m}, 2 \mathrm{H}))$ was scraped off and separated from the decomposition byproducts that remained inside the aluminum foil. GC-MS analysis of the orange crystals revealed that they are $100 \%$ pure 4 (Figure 2). 


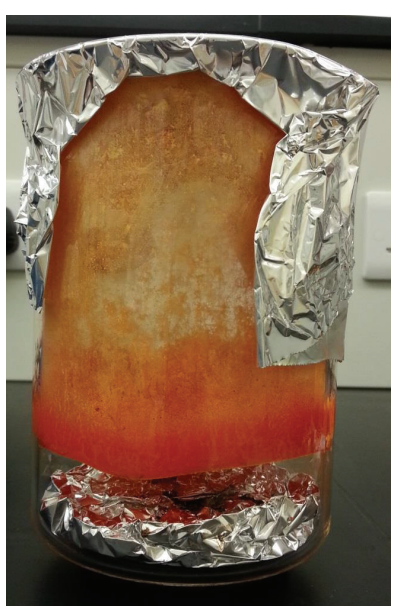

(a)

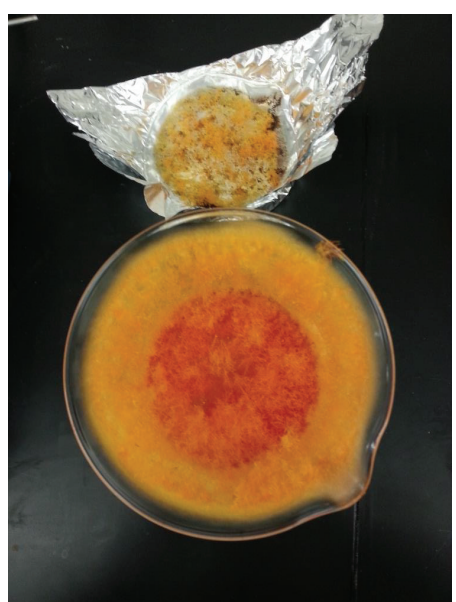

(b)

FIGURE 1: Sublimed 4 after the thermal decomposition of $\mathbf{3}$. Residual byproducts remain inside the aluminum puck at the bottom of the beaker separated from the pure 4 .
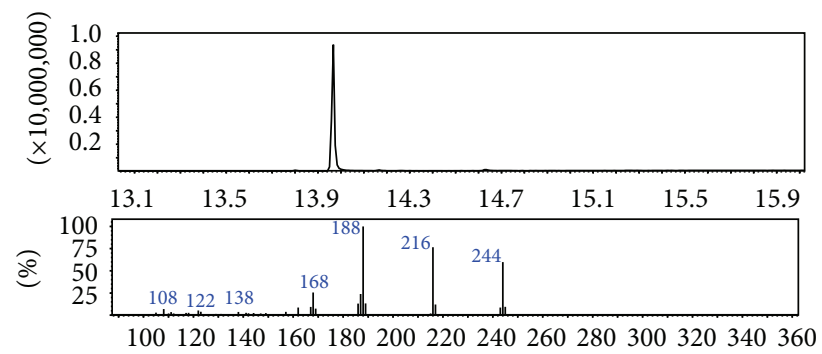

FIGURE 2: GC-MS chromatogram of sublimed 4 (top) giving single peak with retention time of $13.96 \mathrm{~min}$ and an $\mathrm{M}^{+}=244$ with a fragmentation pattern (bottom) corresponding to loss of $\mathrm{CO}$ from the anthraquinone (216 and 188). GC conditions: general-purpose column ( $15 \mathrm{~m}$ long) with a linear velocity flow rate set at $1.2 \mathrm{~mL} / \mathrm{min}$.

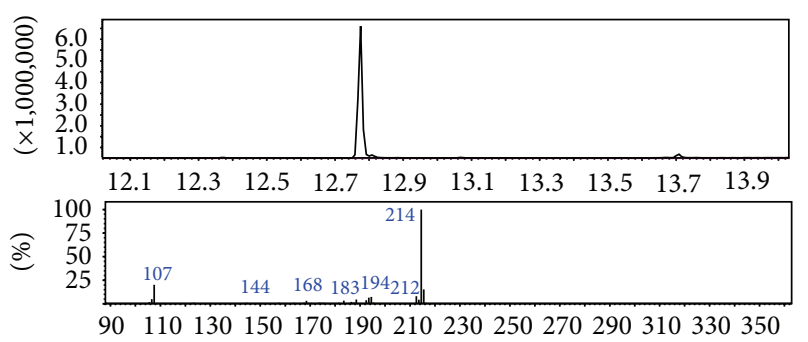

FIGURE 3: GC-MS chromatogram (top) of 1,8-difluoroanthracene 5 $(12.78 \mathrm{~min})$ and the corresponding mass spectrum $\left(\mathrm{M}^{+}=214\right)$ [7].

The yield from the Balz-Schiemann reaction was $47 \%$ of pure 4. It is worth noting that the coprecipitated $\mathrm{NaBF}_{4}$ crystals do not contain any water of hydration, which could reduce the yield. Compound 5 was later synthesized by reducing 4 in aqueous $\mathrm{NH}_{4} \mathrm{OH} / \mathrm{Zn}$ mixture following the reported literature procedure $[7,17]$. GC-MS analysis of crude 5 reveals a very pure product and an $\mathrm{M}^{+}=214$ (Figure 3); thus there was no need for further purification using column chromatography.

\section{Results and Discussion}

Fluoro-substituted aromatic compounds are generally prepared using the Balz-Schiemann reaction [23] in which a diazonium fluoroborate salt, such as $\mathbf{3}$, is thermally decomposed to the organofluorine compound with the copious production of $\mathrm{BF}_{3}$ and dinitrogen gas. The diazonium fluoroborate salt is prepared by precipitating an aqueous solution of a diazonium salt, by adding an inorganic salt containing $\left[\mathrm{BF}_{4}\right]^{-}$ion $\left(\mathrm{NaBF}_{4}\right.$ or $\left.\mathrm{HBF}_{4}\right)$. This method was recently used to synthesize fluorinated derivatives of anthraquinone and anthracene [10]. In general, aromatic diazonium fluoroborates are sparingly soluble in water and thus can be precipitated from aqueous solution with a semiquantitative yield. Thus success of the Balz-Schiemann reaction depends on the ability to precipitate diazonium fluoroborate salt from aqueous solution and washing it free of mineral acids. Unfortunately, partial or complete solubility of some diazonium fluoroborate salts in water will lower the yield. This is one of the reasons why 4 was never synthesized following this rout, since $\mathbf{3}$ is very soluble in water. Utilization of the Balz-Schiemann reaction to synthesize 4 depends on our ability to reduce the solubility of $\mathbf{3}$ in water by utilizing other methods to precipitate it out without hydrolyzing the diazonium ion in the process.

In an effort to reduce the water solubility of $\mathbf{3}$ and force it to precipitate out, 2 was diazotized in $50 \% \mathrm{HBF}_{4}$ saturated with $\mathrm{NaBF}_{4}$; unfortunately this proved unsuccessful and did not motivate 3 to crash out of solution. In an alternative method, we precipitated 3 using a water-soluble organic solvent that does not decompose the diazonium derivative. After several trials we found that isopropanol was the best solvent to quantitatively precipitate out 3 along with $\mathrm{NaBF}_{4}$ without hydrolyzing the diazonium ion. Other water-soluble organic solvents such as methanol or ethanol caused partial dissolution of 3 .

To test the generality of the coprecipitation method, we prepared 1,4-difluoro-9,10-anthraquinone with 39\% yield 
TABLE 1: Different fluoroanthraquinones prepared by the isopropanol precipitation method.

\begin{tabular}{lccc}
\hline Compound & Overall yield after decomposition (\%) & Lit. yield (\%) [10] & Purity by GC-MS \\
\hline 1-Fluoro-9,10-anthraquinone & 82 (sublimed) & 65 & $98 \%$ \\
2-Fluoro-9,10-anthraquinone & 81 (sublimed) & 69 & $98 \%$ \\
2,6-Difluoro-9,10-anthraquinone & 49 (sublimed) & 38 & $95 \%$ \\
\hline
\end{tabular}

${ }^{*}$ The overall yield was calculated based on the amine-anthraquinone starting material yielding the fluoroderivative.

following the same method of coprecipitating the watersoluble diazonium fluoroborate salt with isopropanol. In Table 1 we report the synthesis and yield of various fluorosubstituted anthraquinones prepared by isopropanol precipitation, followed by the Balz-Schiemann reaction.

\section{Conclusion}

Using isopropanol to precipitate the water-soluble diazonium fluoroborate salt 3 followed by thermal decomposition to yield the fluoroderivative is a greener alternative to using concentrated $\mathrm{H}_{2} \mathrm{SO}_{4}$, CsF, or dry DMSO for a Cl-F halogen exchange which is highly susceptible to the presence of water and requires a great deal of workup. By following the isopropanol precipitation method we forgo the concern with ultra-anhydrous conditions of the reagents and column chromatography to purify the final product. This method is general enough to be used for the synthesis of other fluoroanthraquinones with the added advantage of a higher yield.

\section{Competing Interests}

The author declares that they have no competing interests.

\section{Acknowledgments}

Rabih O. Al-Kaysi acknowledges the support of King Abdullah International Medical Research Center (KAIMRC) through Grants RC10/104.

\section{References}

[1] R. Filler, "Organofluorine chemicals and their industrial applications (Banks, R. E., ed.)," Journal of Chemical Education, vol. 58, no. 1, p. A29, 1981.

[2] I. Ojima and T. Taguchi, Fluorine in Medicinal Chemistry and Chemical Biology, Wiley Online Library, 2009.

[3] F. M. D. Ismail, "Important fluorinated drugs in experimental and clinical use," Journal of Fluorine Chemistry, vol. 118, no. 1-2, pp. 27-33, 2002.

[4] C. Isanbor and D. O'Hagan, "Fluorine in medicinal chemistry: a review of anti-cancer agents," Journal of Fluorine Chemistry, vol. 127, no. 3, pp. 303-319, 2006.

[5] S. Purser, P. R. Moore, S. Swallow, and V. Gouverneur, "Fluorine in medicinal chemistry," Chemical Society Reviews, vol. 37, no. 2, pp. 320-330, 2008.

[6] G. A. Olah, R. D. Chambers, and G. S. Prakash, Synthetic Fluorine Chemistry, World Scientific, 1992.
[7] M. W. Haenel, S. Oevers, J. Bruckmann, J. Kuhnigk, and C. Krüger, "Facile syntheses of 1,8-bis(diphenylphosphino)anthracene and 1,8-bis(dimethylamino)anthracene by nucleophilic substitution of 1,8-difluoroanthracene," Synlett, no. 3, pp. 301303, 1998.

[8] E. E. L. Tanner, R. R. Hawker, H. M. Yau, A. K. Croft, and J. B. Harper, "Probing the importance of ionic liquid structure: a general ionic liquid effect on an SNAr process," Organic \& Biomolecular Chemistry, vol. 11, no. 43, pp. 7516-7521, 2013.

[9] M. Matsui, S. Taniguchi, M. Suzuki, M. Wang, K. Funabiki, and H. Shiozaki, "Dyes produced by the reaction of 1,2,3,4tetrafluoro-9,10-anthraquinones with bifunctional nucleophiles," Dyes and Pigments, vol. 65, no. 3, pp. 211-220, 2005.

[10] L. Zhu, F. Tong, C. Salinas et al., "Improved solid-state photomechanical materials by fluorine substitution of 9-anthracene carboxylic acid," Chemistry of Materials, vol. 26, no. 20, pp. 6007-6015, 2014.

[11] A. Gouloumis, R. C. Lawson, P. Vázquez, L. Echegoyen, and T. Torres, "Synthesis and electrochemical switching of a dianthraquinone cryptand and related anthraquinone-diazacrown ether oligomers," Tetrahedron, vol. 58, no. 5, pp. 961-966, 2002.

[12] L. Echegoyen, Y. Hafez, R. C. Lawson, J. de Mendoza, and T. Torres, "Efficient synthesis of alkoxyanthraquinones from fluoroanthraquinones and their preliminary electrochemistry," The Journal of Organic Chemistry, vol. 58, no. 8, pp. 2009-2012, 1993.

[13] L. Bini, C. Müller, J. Wilting, L. Von Chrzanowski, A. L. Spek, and D. Vogt, "Highly selective hydrocyanation of butadiene toward 3-pentenenitrile," Journal of the American Chemical Society, vol. 129, no. 42, pp. 12622-12623, 2007.

[14] M. W. Haenel, S. Oevers, K. Angermund, W. C. Kaska, H.-J. Fan, and M. B. Hall, "Thermally stable homogeneous catalysts for alkane dehydrogenation," Angewandte Chemie-International Edition, vol. 40, no. 19, pp. 3596-3600, 2001.

[15] P. E. Romero, M. T. Whited, and R. H. Grubbs, "Multiple $\mathrm{C}-\mathrm{H}$ activations of methyl tert-butyl ether at pincer iridium complexes: synthesis and thermolysis of $\operatorname{Ir}(\mathrm{I})$ fischer carbenes," Organometallics, vol. 27, no. 14, pp. 3422-3429, 2008.

[16] J. S. Ritch, D. Julienne, S. R. Rybchinski, K. S. Brockman, K. R. D. Johnson, and P. G. Hayes, "Secondary diphosphine and diphosphido ligands: synthesis, characterisation and group 1 coordination compounds," Dalton Transactions, vol. 43, no. 1, pp. 267276, 2014.

[17] J. Hu, H. Xu, M.-H. Nguyen, and J. H. K. Yip, "Photooxidation of a platinum-anthracene pincer complex: formation and structures of $\mathrm{Pt}^{I I}$-anthrone and -ketal complexes," Inorganic Chemistry, vol. 48, no. 20, pp. 9684-9692, 2009.

[18] A. P. Krapcho, Z. Getahun, M. P. Hacker, and J. J. McCormack, "Synthesis and antitumor evaluations of 1, 8-bis[(2-aminoethyl) amino] anthracene-9,10-diones," Bulletin of the Chemical Society of Ethiopia, vol. 2, no. 2, pp. 69-72, 1988.

[19] A. G. Hoeschet, Patent 755668, Aug. 22,1956. 
[20] G. Yamamoto and M. Oki, "Restricted rotation involving the tetrahedral carbon. LX. peri-substituent effect on the rotational barrier of the 9-methyl group in several triptycene derivatives," Bulletin of the Chemical Society of Japan, vol. 63, no. 12, pp. 35503559, 1990.

[21] G. Yamamoto and M. Oki, "Restricted rotation involving the tetrahedral carbon. XXXV. Stereodynamics of 9-(3,5dimethylbenzyl)triptycene derivatives," Bulletin of the Chemical Society of Japan, vol. 54, no. 2, pp. 473-480, 1981.

[22] A. Dahan, T. Ashkenazi, V. Kuznetsov et al., "Synthesis and evaluation of a pseudocyclic tristhiourea-based anion host," The Journal of Organic Chemistry, vol. 72, no. 7, pp. 2289-2296, 2007.

[23] H. Suschitzky, Advances in Fluorine Chemistry, vol. 4, Academic Press, New York, NY, USA, 1965. 

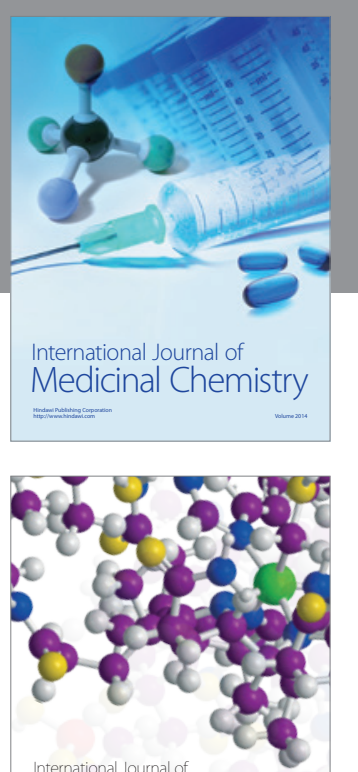

Carbohydrate Chemistry

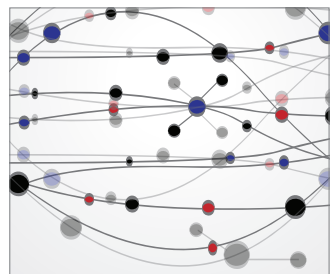

The Scientific World Journal
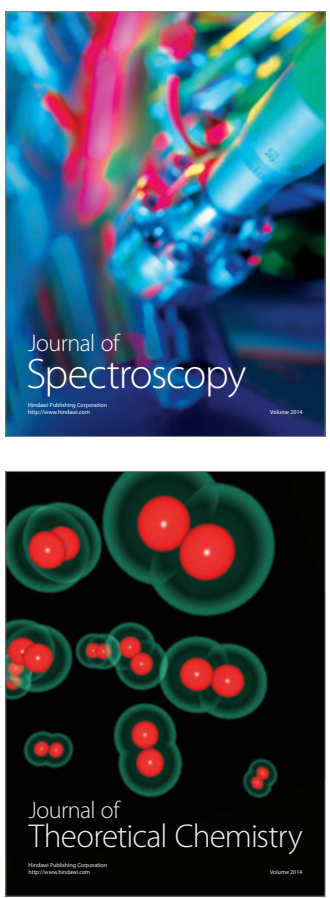
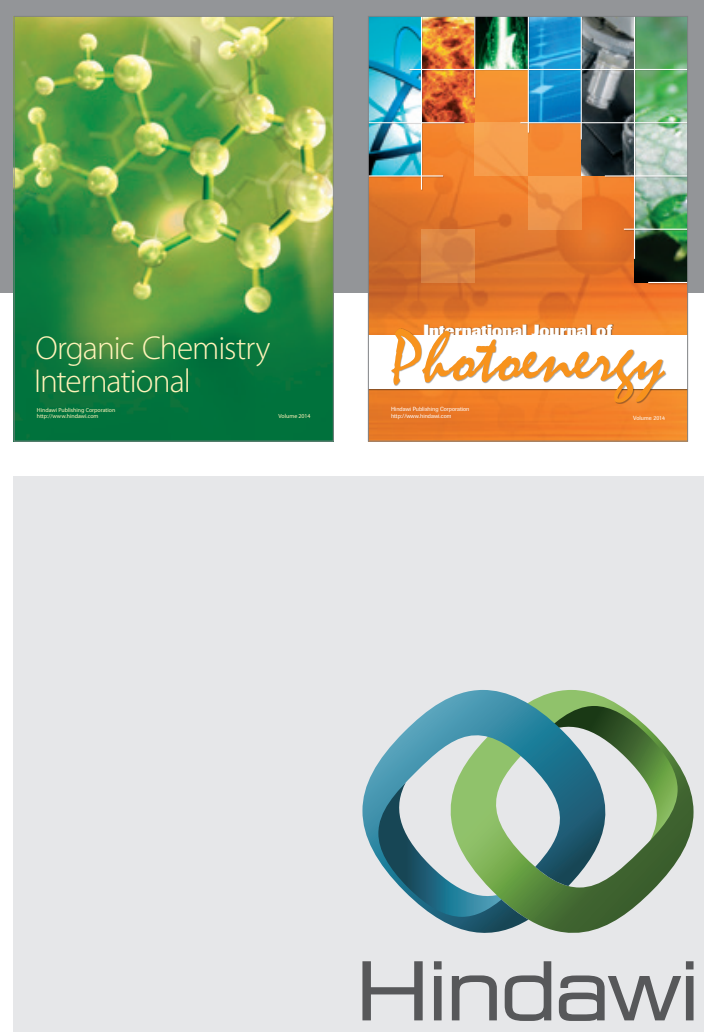

Submit your manuscripts at

http://www.hindawi.com

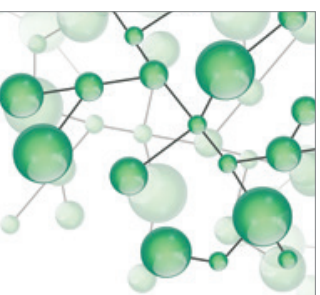

International Journal of

Inorganic Chemistry

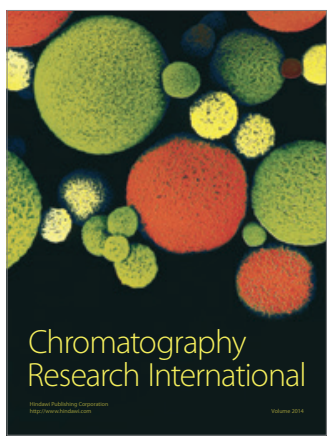

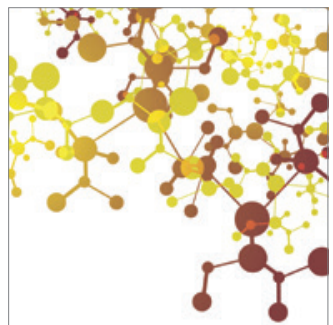

Applied Chemistry
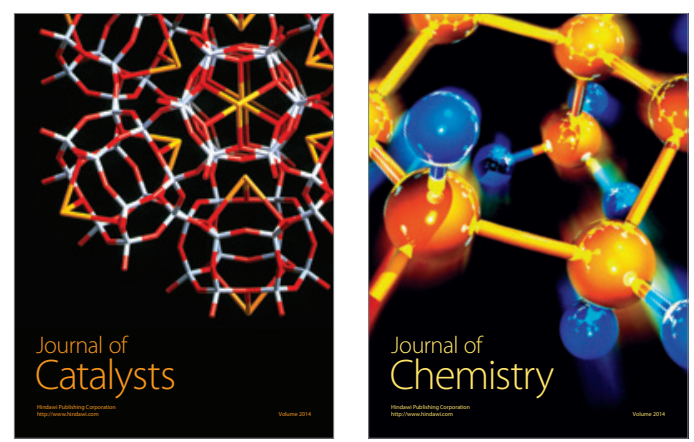
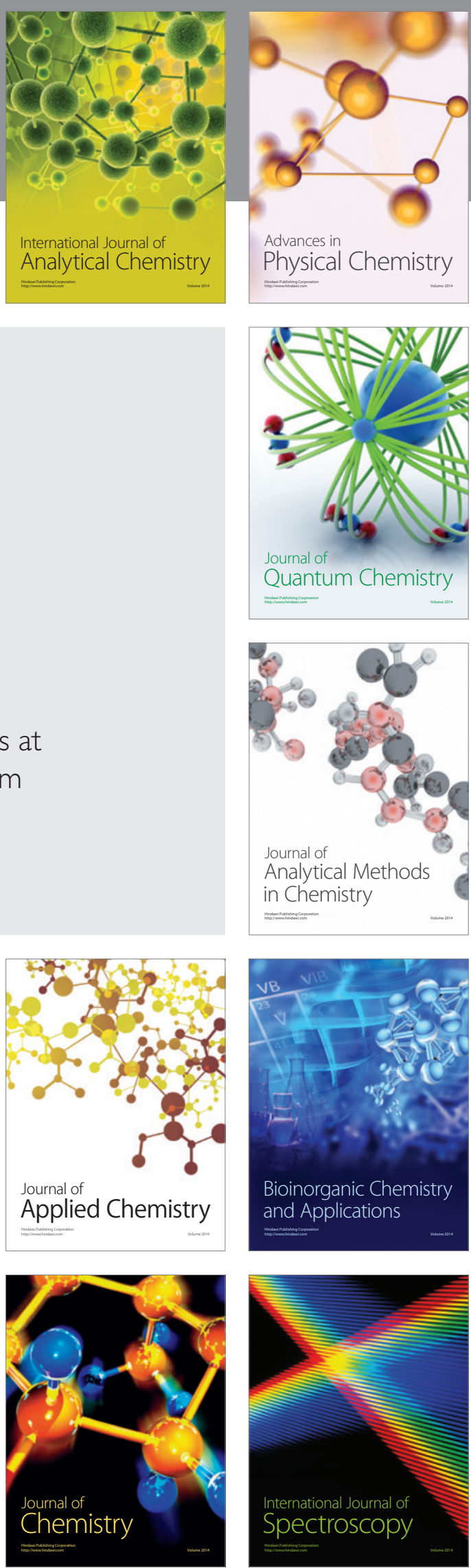